\title{
The Conquest of the Mars
}

\author{
Martin Vlček \\ eFunctionality, Non-profit Research Group, Prague 18200, Czech Republic
}

\begin{abstract}
The exploration of Mars, alike of other planets, would be oriented on a so called on-line tracking. At this approach the development of structural indicators can be followed at time using geological receptors. The principle on what stays this exploration stems from the fact, that a planet is an object, where interactions among its elements are to some extent organized. It means that it goes about an object with a hidden system of control aiming to augment its level of existence and that it is not a "stack of stones". In advance it is necessary to establish some ways-out for the on-line tracking. The first way-out is the fact, that this is an object been monitored, it means a system of organized elements, which has its proper existence and its strategies for a cultivation of this existence. These elements are primarily atoms and for a monitoring of their activities it is possible to use their complexes, e.g. crystals and of course occasionally higher structures, too. Another way-out is the fact, that elements have their proper satisfaction and successfulness and that on basis of those quantities stays the existence of the overall object. These quantities are for new observable mainly on higher structures.
\end{abstract}

Key words: Planet, Mars, tension, boundary, control, successfulness.

\section{Introduction}

\subsection{Analogy}

The basic properties of every object are derived from the functioning of its control systems. These systems function on different levels with the same logic, even if they are working with different elements. However, goals and means are the same [1, 4-12].

The submitted work also arises from this logic. It uses findings from analogical systems proved by extended calculations [2, 3] and finds and applies them on a planet.

On the other side, the author realizes that, similarly as in mentioned objects, it is necessary to prove them experimentally in a planet, too.

\subsection{The Satisfaction and the Successfulness}

Every system works with two basic quantities describing the state of elements and the state of the whole system-new object. These quantities are defined on the basis of inputting or outputting interactions to an element.

Corresponding author: Martin Vlček, RNDr. et RNDr., Ing. research field: cybernetics.
The satisfaction is a function of the evenness and of an average value of inputting interactions. The successfulness is a function of a sum of outputting interactions.

The satisfaction describes the state when necessary inputs for the existence of an element are stably and reliably supplied. The successfulness describes the involvement of an element into the system, the interest for its activity.

It goes perhaps mainly about a sharing and transfer of relatively most free parts of the structure, it means about different parts of atomic and molecular coatings in the case of a planet and thus inorganic elements of different levels. The character of transferred or shared particles changes if it goes about an ionized or other more free structure.

In the case of a planet, the main problem is to reach a quality of receptor able to follow these activities and to have enough of them.

\subsection{Events}

Throughout a tracking of regularities in so complicated systems as a planet is, it is necessary to work with events. It is not possible to suppose that 
throughout a monitoring of a simple value of successfulness and less so of some simple physical quality attributed to the element and by a correlation of it with other values - a regularity will be discovered. It is necessary to work with information of higher level in these so much rustle systems - with an event.

An event is composed from many, of order of hundreds, partly information which describes primarily states of elements, and this in the connection with other elements, and at time. A convenient mechanism for an evaluation of regularities is the forecasting $[2,3]$.

\section{The Control of a Planet}

\subsection{The Central Axe-The Regeneration}

The basic function of a planet, its central axe, is besides the control of the existence of the planet as whole the regeneration of its elements. It consists in not only the solving of sequels of their activities or changes in the environment, but also in a preparation to them, occasionally in an avoidance of unwanted states.

At the beginning of the system of regeneration stems the Input system as a supplier of material inputs. The Input system is also a source of basic impulses for the next control.

The process of regeneration is in its physical-chemical form controlled through the distribution of energy by the most basic system, later on named TOT.

There are two other higher control systems around the central axe and around its energetic control, too. Both work with the successfulness of elements. The successfulness of individual elements through the regeneration evaluates the system named EM0. The satisfaction of the planet as whole, stemming from the tension on the boundary solves the system named EM1.

\section{Input System}

The Input system represents a relatively autonomous and highly important element in the regulatory structure of the planet. Besides the function connected with an input of particles (these particles are not specified and later on will be named as sub-elements because of an absence of accessible information. It goes e.g. about parts of a molecular coat as is hydrogen or protons in the case of water), it goes about the place of origin of impulses, it means of primarily information about the state of the regeneration, respectively of its suppositions - inputs to be at disposal of the planet.

Impulses from the Input system enter through the way of communicative substructures into the planet and form a gradient in what work higher control systems.

There is a bifurcation of ways in the Input system. The material part continues through the way controlled by physical-chemical procedures into places for the subsequent processing. The signal part enters by the pathway of informative substructures and brings the information concerning the state of individual parts of Input system - the boundary.

In general the Input system is formed from two parts. Both are a source of sub-elements. The first part, later on named the primarily boundary, forms sub-elements through its own sources and this is a classical example of the regeneration. Sub-elements which have been transformed throughout the activity of elements into other sub-elements, are inversely transformed with the use of complexes of elements and the energy into the original sub-elements. A regeneration happens. Its possibilities are limited by inner sources and thus in the case of necessity the second part helps.

The second part of the Input system takes sub-elements from the environment and later on is named as a secondary boundary.

\section{TOT}

\subsection{The Control of the Axe-TOT}

The lowest level of the control of the axe is 
connected with the production and the distribution of the energy. Its particularity is among others in the fact, that it does not stem from the feedback from individual elements executing the regeneration, but from the overall satisfaction of the planet with the regeneration. This control system is later on named TOT.

The role of the TOT is to optimize the process of the regeneration from the energetic point view. It means to evaluate the presence of certain indicators of the state of the regeneration and to react in a manner to advance the overall regeneration. TOT reacts not only on the state of these indicators but on their dynamics, too, when a volatility of a state is just a demonstration of a certain status of the regeneration.

TOT in its activity interconnects the energetic system of the planet and of the elements. It mainly determines the distribution into the consumption and into the reserves, into planet and into elements.

\section{EM0}

\subsection{Regeneration}

The task of the EM0 is to control structures executing the regeneration. It thus does not evaluate the successfulness of the regeneration itself, its final effect. This is made through the TOT, but on the basis of the successfulness of individual participating elements and their complexes optimizes the process of the regeneration itself.

The distribution of the successfulness is found out throughout the aggregation of the information depending on the structure of the regeneration. The control proceeds by maximizing the global successfulness, but at the same time by minimizing its volatility. A higher level of the control-EM1—enters into the process of the aggregation into weights of individual factors and thus allows to execute a regeneration already making provision for expected events.

Besides of the control itself the task of EM0 is to form an overall information about the state of the regeneration as a basis for the holistic evaluation in the process of the control of the planet as whole.

\subsection{Philosophy of the Control of EMO}

The general aim from the point of view of the control is to strengthen areas and elements with a high and volatile demand and vice versa. That is why it is necessary to aggregate incoming information about the successfulness until the ultimate level, to make a comparison and depending on the criterion of a volatile successfulness to intervene. The overall fund for the intervention is distributed throughout the desegregation depending on a relative successfulness and volatility of areas.

At the end of the desegregation, on a local level, a lasting reconstruction happens depending on the intensity of the control, as it responds to the distribution of the successfulness.

\subsection{Reconstruction}

The philosophy of the reconstruction is controlled depending on the successfulness of individual elements. It can be said that the success attracts the reconstruction.

The involvement of new elements or working groups proceeds again depending on the success, in a manner that the new element is involved "beside" the most successful one and copies its interaction. The similar success is then expected.

\section{EM1}

\subsection{The Task of EMI}

The task of EM1 is to control events on the boundary of the planet with the environment. It concerns the boundary primarily and secondly, too.

On the boundary happens a tension when the successfulness of some elements or group of them significantly differs from the others, despite the fact that supplied sub-elements are similar. It is a demonstration of a different interest of the planet for their inputs into the process of the regeneration. 
EM1 uses activities on the secondary boundary for the solution of the tension on the primary boundary and as a working boundary uses its inner virtual boundary, later on named as tertiary one.

\subsection{Principle of the Functioning of EM1}

The general principle of the functioning of EM1 is as follows. A finding out of a maximal, resp. the most important tension on the primary boundary happens throughout the aggregation. The tertiary boundary is used for it. This information is projected through the way of desegregation into the movement, what at this case means a redistribution of intensities of inputs on the secondary boundary among individual parts and sub-elements. Thus the tension on the primary boundary is solved through the reconstruction of the secondary boundary.

It is important that EM1 in its work is controlled by global indicators reflecting the state of the distribution of tension on the primary boundary. It goes about the global tension and satisfaction.

\subsection{The Satisfaction}

The satisfaction of the planet, its main indicator, is a function of the evenness of the primary boundary, it means of the level and volatility at time of the successfulness.

The primary boundary thus thanks to its distribution of the successfulness and its dynamics is a criterion and at the same time the hint how to proceed on. EM1 evaluates on the basis of the distribution of the successfulness the real cause of the problem, of a certain dissatisfaction and chooses the strategy depending on it.

This is the intervention of EM1 in the secondary boundary, which provokes changes in intensities of inputs which in their turn have as a sequel a solution of the dissatisfaction of the planet. Then this controlled change of intensity of interactions is another side of the overall movement of the planet in its material environment.
Throughout its work EM1 has to make provision for the tension on the secondary boundary, it means to do the movement in a manner that a too high tension does not arise, or the tension would be only temporally at the time of the cancelling of the primary tension.

The intervention on the secondary boundary itself is similar as in the case of EM0, thus it is a controlled intervention of the fund depending on the distribution of the successfulness.

\subsection{The Tertiary Boundary}

The work of EM1 consists in a usage of an internal boundary forming a transfer among the primary and secondary boundary.

The tertiary boundary ensures that EM1 realizes in a given moment only one global movement and thus a mutual interference does not happen. Similarly only one the most important criterion is solved at a given moment. This is reached through the fact that the inputting tension, which means a non-corresponding successfulness at similar inputs is aggregated to higher levels and only one global, summarily describing tension is find. Only this tension enters into the "consciousness" and is processed. It means that a movement, a reconstruction of the secondary boundary is found out what will cancel this tension.

It is possible to imagine that local tensions or unevenness of successfulness spreads from different informative sources into the tertiary boundary. These partly information are connected, partly on the basis of the last experience and a complex tension arises. If it is sufficiently strong, it will arrive till the tertiary boundary. The process of the aggregation is not only an interconnection but also a suppression of concurring tensions been not a part of a successively identified global tension.

When the tension enters into the consciousness, it "turns" its course and a process of desegregation starts into a movement. A strengthening of well-tried strategies of identification of the tension, which means 
of procedures used throughout the way "up", is also a part of this maneuver. If throughout a gradual realization of the movement an abolishment of individual tensions will arise, strategies identifying this tension throughout the way up will be strengthened. A feedback is thus used when the success of the identification of a tension is verified throughout its abolishment.

\section{Conclusions}

The submitted paper reflects functioning and observing of a planet as whole on the basis of analogies. It is a way stemming from the fact that a planet exists as an integral object connected by common interests of building parts and solving them.

From the point of view of a human these activities are non-observable, but they are very important for the existence of a planet. The problem with the observation of these small relevancies is in the fact, that it is necessary to have to the disposal a great deal of receptors but mainly, that it is necessary to use new methods of processing.

Nevertheless, the cognition of an object as whole, of a new entity is quite fundamental. Besides the fact that it allows to understand new structures and mainly new logics of their functioning, it will allow to enter into a new level of communication with the observed object and to begin to understand it also by a new way-to let it "narrate about itself".

\section{References}

[1] Vlcek M. 2008. "Functioning of the Object." Unpublished material.

[2] Vlcek, M. 2010. "Movers of the Economy-Model of Interactions." In Proceedings of Developments in Economic Theory and Policy, Bilbao, Spain.

[3] Vlcek, M. 2014. "New Object Discovered through the Stock Market." In Proceedings of International Conference on Economics and Finance Research, Seoul, South Korea.

[4] Vlcek, M. 2015. "Firm without Subordinations." American Journal of Economics 5 (3).

[5] Vlcek, M. 2015. "Plant and Its Control Mechanisms." Journal of Life Sciences 9 (4).

[6] Vlcek, M. 2015. "Society and Its Way to the Happiness." American Journal of Economics 5 (4).

[7] Vlcek, M. 2015. "The Cell and Its Worry about Water." Journal of Cytology \& Histology s3-015.

[8] Vlcek, M. 2015. "Philosophy of the Human Organism." Journal of Health Science 3 (5).

[9] Vlcek, M. 2015. "Object and Its Worry about Existence." Philosophy Study 5 (7).

[10] Vlcek, M. 2015. "A Family-The Tension and the Satisfaction.” Sociology Study 5 (5).

[11] Vlcek, M. 2015. "Relations among American Firms Allow the Rise of a New Object." American Journal of Economics 5 (5).

[12] Vlcek, M. 2015. Structure of the Control of the World. Scholars' Press. 